\title{
ESCENAS MUSICALES TRANSFRONTERIZAS Y TRANSCULTURALES: EL NAPAR-MEX 0 LOS CORRIDOS Y RANCHERAS NAVARROS ${ }^{1}$
}

\section{Maite Aperribay-Bermejo}

Universidad del País Vasco / Euskal Herriko Unibertsitatea (UPV/EHU). Dpto. Didáctica de la Lengua y la Literatura.

Grupo de Investigación REWEST

\section{Resumen}

El presente artículo tiene como objetivo aportar una reflexión en relación con la escena musical del Napar-Mex, en la que corridos y rancheras mexicanas se fusionan con influencias, tradiciones y folklore navarro. Las rancheras y corridos mexicanos forman parte del repertorio musical de cualquier celebración popular en la Comunidad Foral de Navarra. La afición de los navarros por lo mexicano es tal, que hace más de dos décadas dio lugar a la creación de una escena musical propia, conocida como Napar-Mex, estilo musical no comercial y estéticamente heterogéneo que surge de la mezcla entre las rancheras, los corridos y el punk-rock vasco. Tras revisar brevemente la relación histórica entre el pueblo vasconavarro y el mexicano, se muestra el viaje de ida y vuelta que efectúa el corrido, cuyo origen reside en el romance español, y que en las últimas décadas ha efectuado un camino inverso, encontrando su lugar en la escena musical vasconavarra.

\section{Palabras clave: NAPAR-MEX; ESCENAS MUSICALES; TRASVASES CULTU- RALES; CORRIDOS; RANCHERAS}

\section{MUSICAL SCENES ACROSS BORDERS AND CULTURES: NAPAR-MEX, CORRIDOS AND RANCHERAS FROM NAVARRE}

\section{Abstract}

This article aims to provide a reflection on the Napar-Mex music scene, in which Mexican corridos and rancheras merge with influences, traditions and folklore from Navarre. Mexican rancheras and corridos are part of the musical repertoire of any popular celebration in the Foral Community of Navarre. Navarre's fondness for the Mexican is such, that more than two decades ago it gave rise to the creation of its own music scene, known as Napar-Mex, a non-commercial and aesthetically heterogeneous musical style that arises from the mix between rancheras, corridos and Basque punk-rock. After briefly reviewing the historical relationship between the Basque and Navarrese people and the Mexicans, the round trip made by the corrido is shown, whose origin lies in the Spanish romance, and which in recent decades has made an inverse path, finding its place in the Basque-Navarrese music scene.

\section{Keywords: NAPAR-MEX; MUSICAL SCENE; CULTURAL TRANSFERS; CORRIDOS; RANCHERAS}

\footnotetext{
Aperribay-Bermejo, Maite. 2021. "Escenas musicales transfronterizas y transculturales: El Napar-Mex o los corridos y rancheras navarros". AusArt 9 (1): 45-54. DOI: 10.1387/ausart.22561
}

\section{AUSART}




\section{LOS VASCONAVARROS Y MÉXICO: TRASVASES CULTURALES Y ECONÓMICOS}

La relación entre estos pueblos se remonta hasta la época de la conquista. Las primeras regiones en ser conquistadas en el Virreinato de Nueva España, territorio que hoy conocemos como México, fueron la provincia de Nueva Vizcaya y la de Nueva Navarra (San Martín 1989; Orozco 2006). Como afirma Ruiz de Gordejuela, "durante el período colonial (...) miles de vasconavarros se asentaron en América, y en especial en la Nueva España" (2011, 21). La llegada de vasconavarros aumentó en la segunda mitad del siglo XVIII y su presencia "fue determinante en el desarrollo económico del México independiente" (Ruiz de Gordejuela 2012, 13). La emigración se agudizó a comienzos del siglo XIX (Azanza 2004) y la última gran corriente de emigración fue la de los exiliados tras el fin de la Guerra Civil (Kenny et al. 1979).

Es evidente que la relación entre el pueblo vasconavarro y el mexicano no ha sido una relación unidireccional, sino una relación de enriquecimiento e influencia mutua, se daban procesos de encuentro y mezcla cultural en ambos sentidos, y se producían trasvases culturales bidireccionales.

\section{EL CORRIDO, HEREDERO DEL ROMANCE Y LA COPLA}

Con el asentamiento de los conquistadores en los territorios de Nueva España las formas populares orales como el romance encontraron cabida en estos territorios, y con el paso del tiempo dieron lugar al corrido. El corrido surgió en México con la adaptación del romance, la copla y la jácara de origen español (Mendoza 2004; González 2002). Mendoza lo define como "un género épico-lírico-narrativo (...) que relata aquellos sucesos que hieren poderosamente la sensibilidad de las multitudes; por lo que tiene de épico deriva del romance castellano y mantiene (...) su carácter narrativo de hazañas guerreras y combates, creando entonces una historia por y para el pueblo" (2004, IX).

Aurelio González sostiene que "al ser trasplantado a tierras americanas, se enriquece con formas léxicas y topónimos nuevos, (...) contextos locales y referentes históricos novedosos, pero contando la misma historia" $(2002,212)$. 
Afirma que la apertura del romance y su enriquecimiento gracias al contexto local permiten que en ocasiones este se reconvierta en un corrido, "un género literario plenamente constituido con auge tanto entre el público rural como el urbano, y valorado en distintos estratos sociales, en unos casos por su valor noticioso, en otros por su contenido propagandístico o ideológico" (ibíd., 215). Esta transición del romance al corrido hace que se mantengan algunos elementos, siendo el más significativo el de la presencia de personajes transgresores, bandidos o luchadores sociales, cuya caracterización los identifica como héroes de corridos. Reyes (1997) señala que la principal diferencia entre el romance y el corrido está en sus protagonistas, puesto que el romance exalta la vida de un personaje noble, mientras que el corrido narra historias de personajes de origen humilde. Lira-Hernández (2013) afirma que el corrido es una narración oral cuyo contenido puede ser tanto informativo como reivindicativo, que es primordialmente regional o local, que posee una estructura lírica y musical, aunque dicha estructura no es estable.

Se trata de una escena musical cuya expansión geográfica abarca no solo el territorio mexicano, sino que ha rebasado fronteras, llegando, entre otros, a los EE.UU., Chile, Colombia, Brasil, Venezuela y Holanda (Montoya \& Medrano de Luna 2016). Como afirma Mendoza, "en Estados fronterizos del Sur y ciudades donde se le encuentra vivo como manifestación cultural de origen hispánico, ha dado lugar a la creación y derivación de nuevos tipos que muestran ya lineamientos locales" (2004, VIII). Si bien España no se incluye como uno de los países a los que ha llegado la influencia del corrido, el objetivo de este artículo es demostrar que la escena musical conocida como Napar-Mex ha surgido en las últimas décadas tras haber regresado el corrido transformado a sus raíces.

\section{NAPAR-MEX: REINTERPRETACIÓN DE LOS CORRIDOS Y LAS RANCHERAS MEXICANAS}

El interés por las escenas musicales (Straw 1991) estatales ha aumentado, si bien la gran mayoría de los estudios se han centrado en escenas reconocidas como La Movida madrileña y el rock urbano y el heavy (Val 2013), la Onda Layetana en Barcelona (Gómez-Font 2013), el Sabadell Sound de las discotecas de la Costa Mediterránea (Ripollès Iranzo 2011) y el punk (Porras Blanco 2006) y el Rock Radical Vasco (RRV) en el País Vasco y Navarra (Mota Zurdo 
2017; Moso 2013). Hasta el momento la escena musical del Napar-Mex es una escena por estudiar, puesto que no ha sido considerada aún objeto de ningún trabajo de carácter académico.

Bennett define una escena musical como "a particular local setting (...) where a particular style of music has either originated, or has been appropriated and locally adapted" $(2004,223)$. Es un concepto plenamente aceptado para estudiar las prácticas musicales que se producen en un contexto geográfico concreto, o que traspasan esos mismos contextos asentándose en otros espacios geográficos. La idea de espacialidad es fundamental, puesto que Kotarba, Fackler, y Nowotny definen una escena musical como "the geosocial location that provides a stage on which all of the aesthetic, political, social, and cultural features of local music are played out" $(2009,312)$. Hoy en día, "las comunicaciones globales han hecho que lo local se expanda con cierta facilidad más allá de las fronteras" (Val 2013, 44).

Como se ha mencionado anteriormente, los trasvases culturales entre los pueblos mexicano y vasconavarro, que se remontan siglos atrás, siguen teniendo efecto en la cultura y el folklore. A nivel estatal esta influencia ha sido evidente, de la mano, por ejemplo, de Luis Mariano, Rocío Dúrcal, María Dolores Pradera y otros grupos o solistas como el Dúo Gala, Paco Formoso o Bertín Osborne, quienes han popularizado las rancheras y los corridos a nivel estatal.

En el norte de la península el gusto por lo mexicano es notable, sobre todo en la Comunidad Foral de Navarra, donde los corridos mexicanos forman parte del repertorio musical de cualquier celebración popular, en la que se mezclan con estilos más locales como las jotas, con las que guardan parecido a nivel sonoro. En Navarra, numerosos solistas y grupos producen rancheras y corridos desde hace décadas. Cabe mencionar, por ejemplo, a Chus Isaba, el Dúo Monterrey, Mariachi Zacatecas, Trío Jalisco, Trío Veracruz, Chuchín Ibáñez y un largo etcétera. En esta ocasión, sin embargo, nos centraremos en la producción de grupos pertenecientes al estilo denominado Napar-Mex, escena musical de evidente influencia mexicana surgida en Navarra en la década de los noventa. La música producida bajo este estilo es una adaptación popular y local de los corridos y las rancheras mexicanas, y es que, tal y como sostiene Bennett (2004), las escenas y los estilos musicales populares pueden ser reapropiados desde nuevas perspectivas locales, de tal manera que estas escenas sean más significativas para esos nuevos contextos. 
El Napar-Mex es un estilo musical no comercial y heterogéneo con un ámbito de difusión reducido. Se trata en su mayoría de canción protesta, su audiencia es generalmente joven y alternativa, su tono es reivindicativo e informal, y en ocasiones tiene cierta dimensión paródica. Se trata de una escena musical popular, no tiene una pretensión culta ni académica, sino que su objetivo es generalmente el de ser un medio de identificación y, al mismo tiempo, ser un medio de diversión.

Este estilo musical también tiene influencia del Rock Radical Vasco (RRV), una escena musical que surgió a comienzos de los años ochenta (Moso 2013). Bajo esta controvertida etiqueta, con la que muchos grupos no comulgaban, se incluía a grupos como RIP, Eskorbuto, Hertzainak, Cicatriz o los navarros Tijuana in Blue. Esta última banda de punk-rock fue pionera del Napar-Mex. Las letras de Tijuana in Blue reflejaban el descontento de la juventud de la época con la realidad del momento y con la clase política, sus canciones eran reivindicativas y trataban temas como el abuso de la autoridad o la inmigración. En 1985 publicaron la maqueta Directo Barcelona, en la que bajo el título Con dinero y sin dinero, el grupo versionó el clásico El Rey, compuesta por José Alfredo Jiménez y popularizada por cantantes de la talla de Vicente Fernández o María Dolores Pradera (Almeida 2013). El grupo publicó seis discos antes de su disolución en 1991. Ese mismo año su vocalista viajó durante unos meses a México, donde su afición por las rancheras creció mientras residía en Tijuana. A su regreso "trajo consigo unos 80 discos que pincharía de (narco) corrido en las tres temporadas que duró Fiesta Mexicana, celebrado programa de la Eguzki"2 (Las rancheras 2016). Poco después de su regreso fundó Kojón Prieto y los Huajolotes (o Huajalotes), que logró reconocimiento gracias a sus mezclas de punk-rock con rancheras y música mexicana (Porras Blanco 2006).

Como apuntaba Mendoza, las rancheras y corridos han dado lugar a nuevos "lineamientos locales" (2004, VIII), y si bien el estilo lo popularizó el grupo Kojón Prieto, hoy en día, casi treinta años después de su surgimiento, numerosos grupos se incluirían en la escena del Napar-Mex. Entre ellos cabe mencionar a Los Txarros Txilapastrosos, La Mala Pékora, Los Zopilotes Txirriaos o Los Tenampas y El Mariachi. Entre todos estos grupos son los dos últimos los que gozan de mayor popularidad.

Los Zopilotes Txirriaos publicó su primera maqueta, titulada Napar-Mex, en 2009. En 2011 hicieron lo propio con su primer disco, Hijos de la Txingada. Su segundo disco, La Salutxita, vio la luz en 2013, y en 2017 publicaron Entre Atxunes y Culebras. Entre sus creaciones nos encontramos con el Corrido a 
Luzio Urtubia y con una adaptación del popular corrido de La Cucaracha, que pertenecen a su segundo y tercer disco respectivamente.

El primero de los corridos está dedicado a Urtubia, agricultor y albañil navarro que mientras cumplía su servicio militar se dedicó al contrabando. Al ser descubierto desertó y huyó a Francia, donde entró en contacto con el movimiento anarquista. A finales de los setenta puso en jaque al mayor banco del mundo, el First National City Bank of America, estafando veinte millones de dólares con la falsificación masiva de cheques de viaje. Cual Robin Hood moderno, repartió el dinero entre diversas causas sociales, y falsificó documentación para numerosos exiliados españoles, gracias a la cual muchos pudieron huir de la represión franquista (Duva 2007).

Salvando las distancias con los corridos de héroes o bandoleros mexicanos, el Corrido a Luzio Urtubia guarda semejanzas con conocidos corridos mexicanos. Dentro de los asuntos que trata el corrido, Mendoza menciona, entre otros, los corridos de hechos históricos o corridos revolucionarios, entre los que distingue los políticos y de carácter lírico, los de valientes o los de bandoleros y afirma que los "políticos y de carácter lírico son aquellos en que los hombres ponen de por medio sus ideales" (2004, XXXVI). El Corrido a Luzio Urtubia habla de "un hombre libre y sin dueño (...). Su suerte fue nacer pobre y no guardarle respeto a todo lo establecido por los jueces y el gobierno, la iglesia y las propiedades". Los corridos de valientes aglutinan "los hechos de individuos que despliegan sus facultades desafiando los peligros, las persecuciones y la muerte" (Mendoza 2004, XXXVII). Como refleja el corrido de Los Zopilotes, Urtubia se dedicaba al contrabando y al estraperlo, y desafió nada más y nada menos que al mayor banco del mundo. Mendoza también enumera los corridos de bandoleros, que relatan "las hazañas de una multitud de hombres (...) rebeldes a todos los gobiernos, perseguidos en calidad de bandoleros, forman un grupo aparte. Los hubo (...) que robaban a los ricos para dar a los pobres" (2004, XXXVII-XXXVIII). Y qué mayor bandido que Urtubia, que "dio la mano al refugiado y a la gente perseguida", robando a un gran banco para destinar el dinero a causas de índole social.

Aunque este corrido no respete la forma clásica de los corridos, puede considerarse una reinterpretación de estos. Para comenzar el texto dice "quiero echarles un corrido", lo que resulta ser una clara marca de oralidad. En lo que al contenido se refiere, cuenta la historia de un bandolero, un contrabandista revolucionario y libertario que se dedicaba al estraperlo y al mismo tiempo ayudaba a los exiliados, guardando cierta similitud con los corridos políticos, 
de valientes o de bandoleros. Otro de los puntos en común con los corridos sería función informativa, puesto que como afirma Henestrosa (1977, 10), "el corrido es el vehículo de que el pueblo se vale no sólo para expresarse: es también su órgano periodístico", lo cual también se cumple en este caso, ya que el corrido recoge la vida de Urtubia e informa al público de sus hazañas.

Otro de los grupos que actualmente gozan de mayor popularidad son Los Tenampas y El Mariachi. Una de sus canciones se titula Un charro en San Fermín (2017). Refleja la alegría que las rancheras provocan en la población local: "Desde Tijuana, una mañana / llegó este charro a San Fermín / era un tornado, blanco y colorado / esa Pamplona que antes fue gris". La ranchera nos informa de que "el Mariachi detiene su chamba / en la Plaza del Castillo", que es el centro de la capital navarra. El estribillo recoge dos voces, una de las cuales canta la canción de San Fermín mientras que otra de las estrofas centrales reza "yo soy el Rey y tú la princesa / y nos fuimos juntos por la Estafeta", haciendo referencia a la que probablemente sea la arteria más conocida de la capital navarra. Como no podía ser de otra manera, la ranchera termina diciendo que "nos soltamos lentamente los pañuelos del cuello / Hasta el próximo siete de julio", haciendo clara alusión al "Pobre de mí".

Su letra refleja el amor de los navarros por San Fermín y en esta composición se aprecia la clara mezcla del folklore mexicano, puesto que el grupo conserva la estética mexicana de los mariachis y los trajes charros, pero la impregnan de símbolos navarros como San Fermín. La letra sitúa la canción en las calles de la capital navarra y en el videoclip las peñas transitan junto a los mariachis por parte del recorrido de los encierros, concluyendo en la céntrica y popular Plaza del Castillo, en una suerte de "Pobre de mí", la tradicional despedida de las fiestas en la que los pamploneses se sueltan el nudo del pañuelo.

\section{CONCLUSIONES}

Los años ochenta fueron testigos del surgimiento de una enorme cantidad de estilos y escenas musicales a nivel estatal, fruto todas ellas del cambio que estaba atravesando el país, y la región vasconavarra no fue una excepción. En esa década surgió el heterogéneo Rock Radical Vasco, que sin duda ha influenciado otras escenas musicales, entre las que podemos mencionar el Napar-Mex, que puede ser considerada a su vez una escena local de la 
música mexicana. Esta producción musical estaba vinculada a la idea de cambio, era transgresora, políticamente comprometida, generalmente autogestionada y alternativa. La difusión de esta música tuvo que buscar sus propios espacios dentro de la ciudad y la comunidad. En ese escenario alternativo se gestó la fundación del grupo Tijuana in Blue, que fueron los primeros en utilizar la etiqueta Napar-Mex.

El Napar-Mex es una reinterpretación de la música mexicana desde la perspectiva navarra, y por tanto una mezcla de culturas que recrea diferentes identidades. El Napar-Mex es en cierto modo heredero del corrido mexicano, puesto que, aunque generalmente no se observe el esquema formal tradicional, tienen características comunes. La principal es su carácter popular, ya que en ambos casos se trata de composiciones narrativas que documentan la historia de las comunidades, dando voz al pueblo y creando un sentimiento de unidad. El Napar-Mex es una escena musical que goza de buena aceptación en la zona vasconavarra, y que parece tener futuro, puesto que a menudo muestra el descontento ciudadano, convirtiéndose en canción protesta. Quizá por encima de cualquier otra característica, y sobre todo en el caso del desenfadado Napar-Mex, prevalece el objetivo de celebración y el divertimento.

\section{Referencias bibliográficas}

Almeida Pérez, Jaime. 2013. "José Alfredo Jiménez, el rey de la canción ranchera". Declaraciones de Jaime Almeida. Secretaría de Cultura Gobierno de México, 13 nov. https://www. gob.mx/cultura/prensa/jose-alfredo-jimenez-el-rey-de-la-cancion-ranchera-jaime-almeida?state=published

Azanza López, José Javier. 2004. "Aproximación a la arquitectura de los americanos en Navarra”. Príncipe de Viana 65(232): 421-74

Bennett, Andy. 2004. "Consolidating the music scenes perspective". Poetics 32(3-4): 223-34. https://doi.org/10.1016/j.poetic.2004.05.004

Calvo Ridruejo, Carmelo. 1984. "Clausuradas dos emisoras libres en Pamplona". El País, 23 marzo. https://elpais.com/diario/1984/03/23/radiotv/448844404_850215.html

Duva Milán, Jesús. 2007. "El albañil que estafó al mayor banco del mundo". El País, 8 nov. https://elpais.com/diario/2007/11/11/eps/1194765361_850215.html

García Gil, Samuel, Salvador Gómez García \& Itziar Reguero Sanz. 2018. "Espacios alternativos de libertad durante la Transición: Breve historia de las radios libres en España (1976-1983)". Revista Latina de Comunicacion Social 73: 1179-210. https://doi.org/10.4185/ RLCS-2018-1302

Gómez-Font, Álex. 2013. "Cataluña y el rock". En Rock around Spain: Historia, industria, escenas y medios de comunicación, editado por Kiko Mora \& Eduardo Viñuela, 123-38. Leida: Universitat de Lleida 
González, Aurelio. 2002. "Del Romance al Corrido: Estilo, temas y motivos." En Lenguajes de la tradición popular: Fiesta, canto, música y representación, editado por Yvette Jiménez de Báez, 207-20. Ciudad de México: El Colegio de México

Henestrosa Morales, Andrés. 1977. Espuma y flor de corridos mexicanos. Ciudad de México: Porrúa

Kenny, Michael et al. 1979. Inmigrantes y refugiados españoles en México: Siglo XX. Ciudad de México: Centro Investigaciones Superiores del INAH

Kotarba, Joseph A., Jennifer L. Fackler \& Kathryn M. Nowotny. 2009. "An ethnography of emerging Latino music scenes". Symbolic Interaction 32 (4): 310-33. https://doi.org/10.1525/ si.2009.32.4.310

Lira-Hernández, Alberto. 2013. "El corrido mexicano: Un fenómeno histórico-social y literario". Contribuciones desde Coatepec 24: 29-43

Mendoza Gutiérrez, Vicente Teódulo. (1954) 2004. El corrido mexicano. Ciudad de México: Fondo de Cultura Económica

Montoya Arias, Luis Omar \& Gabriel Medrano de Luna. 2016. La música norteña mexicana. Guanajuato: Universidad de Guanajuato

Moso Gil, Roberto. 2013. “El rock radikal vasco: Ruido y rabia en la 'Zona Especial del Norte'”. En Rock around Spain: Historia, industria, escenas y medios de comunicación, editado por Kiko Mora \& Eduardo Viñuela. Lleida: Universitat de Lleida

Mota Zurdo, David. 2017. Los 40 radikales: La música contestataria vasca y otras escenas musicales; Origen, estabilización y dificultades (1980-2015). Bilbao: Beta III Milenio

Orozco Linares, Fernando. 2006. Historia de México: De la época prehispánica a nuestros días. Ciudad de México: Panorama

Porras Blanco, Juan. 2006. Negación punk en Euskal Herria. Tafalla: Txalaparta

Reyes Hernández, Judith. 1997. El corrido: Presencia del juglar en la historia de México. Texcoco de Mora: Universidad Autónoma Chapingo

Ripollès Iranzo, Joan. 2011. "Sabadell Sound: Ni inglés, ni italiano, sino todo lo contrario". Rinconete, web Centro Virtual Cervantes, 11 ago. https://cvc.cervantes.es/el_rinconete/ anteriores/agosto_11/11082011_01.htm

Ruiz de Gordejuela Urquijo, Jesús. 2011. Vivir y morir en México: Vida cotidiana en el epistolario de los españoles vasconavarros 1750-1900. Vitoria-Gasteiz: Nuevos Aires

— . 2012. Vasconavarros en México. Prólogo de María del Carmen Saucedo. Madrid: LID

San Martín Ortiz de Zarate, Juan. 1989. "Francisco de Ibarra, conquistador de Nueva Vizcaya y fundador de la ciudad de Durango, en México". Euskera 34 (1): 299-304

Straw, Will. 1991. "Systems of articulation logics of change: Communities and scenes in popular culture". Cultural Studies 5 (3): 368-88. https://doi.org/https://doi. org/10.1080/09502389100490311

Val, Fernán del. 2013. "El rock español en los 80: Del underground a la institucionalización". En Rock around Spain: Historia, industria, escenas y medios de comunicación, editado por Kiko Mora \& Eduardo Viñuela, 39-52. Lleida: Universitat de Lleida 
Notas

${ }^{1}$ El presente trabajo se ha realizado bajo el auspicio del grupo de investigación REWEST, de la Universidad del País Vasco UPV/EHU, y ha sido financiado por el Gobierno Vasco (IT102616) y por el Ministerio de Ciencia, Innovación y Universidades (PGC2018-094659-B-C21).

${ }^{2}$ Eguzki Irratia (Radio Sol) es una emisora de radio comunitaria pamplonesa que comenzó sus emisiones a comienzos de los años ochenta. Es una emisora libre, autogestionada y alternativa, que aboga por la libertad de expresión y comunicación y que ha sobrevivido a numerosos cierres a lo largo de su historia (Calvo Ridruejo 1984). Eguzki Irratia y otras muchas radios libres, junto con los populares fanzines de la época, propiciaron la contrainformación al movimiento de resistencia juvenil de los años ochenta y noventa, pasando a ser la expresión alternativa a los medios de comunicación oficiales (García Gil, Gómez García \& Reguero Sanz 2018). 